\title{
Correlation of microbiota in the gut of fish species and water
}

\author{
Ao Zeng ${ }^{1} \cdot$ Kai Tan $^{2} \cdot$ Ping Gong $^{1} \cdot$ Ping Lei $^{1} \cdot$ Zhaohui Guo $^{1} \cdot$ Shengping Wang ${ }^{1} \cdot$ Shufeng Gao ${ }^{1} \cdot$ Yinghua Zhou ${ }^{1}$. \\ Yan Shu ${ }^{1} \cdot$ Xiaoling Zhou ${ }^{1}$. Dong Miao ${ }^{1} \cdot$ Fajiao Zeng ${ }^{1} \cdot$ Huizhi Liu ${ }^{1}$ (i)
}

Received: 3 July 2020 / Accepted: 30 September 2020 / Published online: 12 October 2020

(c) The Author(s) 2020

\begin{abstract}
To analyze the intestinal microbiota diversity of several important economic fishes in the Loudi area and its correlation with the microbiota of water environment, the high-throughput sequencing based on the bacteria $16 \mathrm{~S}$ rRNA was used to analyze the intestinal microbiota diversity in fish intestines and water. The results revealed that half of the OTUs in the water sample could be detected in the fish intestine, the proportion of shared OTUs in the intestines of Hypophthalmichthys molitrix and water samples was only $22 \%$, and the unique OTU in the LC group was relatively the highest in the fish intestinal group. It can be seen from the analysis in NMDS analysis, the distance between Hypophthalmichthys molitrix group and water group is relatively farthest. Ctenopharyngodon idellus has the highest microbiota richness and diversity $(P<0.05)$, while the water samples have the lowest microbiota richness $(P<0.05)$. Firmicutes, Methylocaldum and Bacillus are the prevalent taxonomic unit in the Aristichthys nobilis and Carassius auratus groups, Anaerospora is the prevalent genera in the Hypophthalmichthys molitrix group, Proteobacteria and Cyanobacteria have a high relative abundance ratio in the Ctenopharyngodon idellus group, and the prevalent taxonomic unit in the water sample group are Phenylobacterium, Bacteroidetes and Actinobacteria. In conclusion, fish species have different prevalent microbiota. There are a strong correlation between fish intestinal microbiota and the water environment, and the fish with a weak correlation is Hypophthalmichthys molitrix. Results of this study will contribute to the prevention and treatment of fish diseases and the fish ecological culturing in Loudi area.
\end{abstract}

Keywords Fish species $\cdot$ Gut microbiota $\cdot 16 \mathrm{~S}$ rRNA $\cdot$ Loudi

\section{Introduction}

The intestinal microbiota of fish are much more simpler than mammals (Mitra et al. 2014; Okadinya et al. 2013). Similarly, due to the limitations of fish physiological conditions, in which the evolution of the intestine of fish is not complete, the digestive enzymes secreted by intestinal microorganisms and the regulating functions of intestinal microorganisms on fish immunity are particularly important. Intestinal physiological microbiota, fish body and the surrounding water environment constitute a ecosystem, which is interdependent

Huizhi Liu

17352879823@163.com

1 Department of Animal Nutrition, Hunan Institute of Microbiology, No 18 Xinkaipu Road, Changsha 410009, Hunan, China

2 College of Fisheries and Life Science, Shanghai Ocean University, Shanghai 201306, China and mutually restricted. The balance of this system is the key factor to maintain the health of fish.

The gut microbiota of fish is affected by a variety of factors. Among these, the main factors are the type of fish and the waters it inhabits, while other factors include the bait, development stage, water temperature and physiological conditions (Jiang et al. 2016). At present, there have been many reports on the microbiota composition of freshwater fish. Different from the mammalian intestinal microbiota, which are mainly dominated by bifidobacteria and lactobacillus, the prevalent intestinal microbiota of fish has exhibited huge differences, and even the intestinal prevalent microbiota of the same fish differs in different regions (Nilsson et al. 2016; Chen et al. 2015). Therefore, the study of fish intestinal microorganisms should be systematic or qualitatively researched in fixed culturing conditions.

Pond intensive aquaculture has a short cycle and high yield, which is the main mode of aquaculture in China. However, this model has a high culturing density, and a large amount of bait and medicine are used, which has a great 
negative impact on disease outbreaks and the surrounding environment ( $\mathrm{Li}$ et al. 2010). At this stage, the microecosystem comprise of the fish gut, fish body and the surrounding water environment in the Loudi area, which is already very fragile. Therefore, it is necessary to study the gut microbiota of fish in different environmental conditions. The present study aims to analyze the intestinal bacterial diversity of several important economic fishes in the Loudi area and their correlation with the bacterial structure of the water environment, to provide basic data for the prevention and treatment of fish diseases, the use of microbial additives, and the fish ecological culturing in this area.

\section{Materials and methods}

\section{Collection of samples}

The four kinds of experimental fishes included Hypophthalmichthys molitrix, Aristichthys nobilis, Ctenopharyngodon idellus and Carassius auratus. The water samples were collected from the same pond at the aquaculture base of Loudi Institute of Fishery Science. The pond has a water depth of $2.0 \mathrm{~m}$ and an area of $1.5 \mathrm{hm}^{2}$. Commercial fish feed (Grass Carp 707 series, Haida Group) was fed for two times a day (08:00 and 15:00 h). The fish and water samples were taken at $07: 00 \mathrm{~h}$ on June 25,2019 . The water temperature was $27.8^{\circ} \mathrm{C}, \mathrm{pH}$ was 6.95 , and the dissolved oxygen was $3.35 \mathrm{mg} \mathrm{L}^{-1}$. In the tira net catch, three healthy fishes of the same size were randomly selected from each type for weighing, which included Ctenopharyngodon idellus $0.60 \mathrm{~kg}$, Carassius auratus $0.21 \mathrm{~kg}$, Hypophthalmichthys molitrix $0.35 \mathrm{~kg}$, and Aristichthys nobilis $0.31 \mathrm{~kg}$. At the same time, water samples were collected at $1.0 \mathrm{~m}$ below the water surface from three randomly selected points in the fish pond. Then, these samples were immediately ice-bathed and shipped back to the laboratory.

\section{Extraction of contents and collection of bacteria in water samples}

After the fish samples were returned to the laboratory, fish samples were anesthetized with eugenol $(1: 10,000)$, and the surface of the fish was successively rinsed with sterile water and $70 \%$ ethanol. Then, the contents of the foregut section of all samples were aseptically collected in a sterile operation box. Twelve intestinal contents were sampled as Hypophthalmichthys molitrix (LC1-LC3), Aristichthys nobilis (YC1-YC3), Ctenopharyngodon idellus (CC1-CC2), and Carassius auratus (JC1-JC3).

After the water sample was transported back to the laboratory, the water sample was filtered with a sterile fil$\operatorname{ter}(0.2 \mu \mathrm{m})$ under a sterile environment. Each sample was filtered by $2 \mathrm{~L}$, and the filter membrane was stored for future use. The sample numbers were SS, SZ and SX.

\section{Extraction of DNA}

The water environmental DNA and content DNA were extracted using a Tiangen stool DNA extraction kit (TIANamp Stool DNA Kit). The specific operations were based on the kit instructions. The extracted DNA was analyzed by agarose gel electrophoresis, and the concentration and purity of the total DNA were detected using an ultra-micro ultraviolet spectrophotometer. Finally, the DNA was stored at $-20{ }^{\circ} \mathrm{C}$ until use.

\section{PCR amplification and preparation of the sequencing library}

The total sample DNA was used as the template for the PCR amplification of the bacterial $16 \mathrm{~S}$ rRNA gene in the V3+V4 region. The $\mathrm{V} 3+\mathrm{V} 4$ variable region common primer was synthesized by Shanghai Personal Biotechnology Co., Ltd. The primer sequence was 338F: 5'-ACTCCTACGGGAGGC AGCA-3' and 806R: 5'-GGACTACHVGGGTWTCTAAT3'. PCR amplification system: $25 \mu \mathrm{L}: 5 \times$ reaction buffer $5 \mu \mathrm{L}, 5 \times \mathrm{GC}$ buffer $5 \mu \mathrm{L}$, dNTP $(2.5 \mathrm{mM}) 2 \mu \mathrm{L}$, forward primer $(10 \mu \mathrm{M}) 1 \mu \mathrm{L}$, reverse primer $(10 \mu \mathrm{M}) 1 \mu \mathrm{L}$, DNA template $2 \mu \mathrm{L}, \mathrm{ddH}_{2} \mathrm{O} 8.75 \mu \mathrm{L}$, and Q5 DNA polymerase $0.25 \mu \mathrm{L}$. Amplification parameters: initial denaturation at $98{ }^{\circ} \mathrm{C}$ for two minutes, denaturation at $98{ }^{\circ} \mathrm{C}$ for $15 \mathrm{~s}$, annealing at $55^{\circ} \mathrm{C}$ for $30 \mathrm{~s}$, extension at $72{ }^{\circ} \mathrm{C}$ for $30 \mathrm{~s}$, and final extension at $72{ }^{\circ} \mathrm{C}$ for $5 \mathrm{~min} ; 29$ cycles. The PCR products were detected by agarose gel electrophoresis and purified using the agarose gel recovery kit (Shao et al. 2020). The fluorescence reagent used was the quant-it PicoGreen dsDNA Assay Kit, and the quantitative instrument performed using a microplate reader (BioTek, FLx800).

The sequencing library was prepared according to the instructions of the TruSeq Nano DNA LT Library Prep Kit (Illumina). The library concentration was $>2 \mathrm{nM}$. The amplified V3 + V4 variable region of the 16S rRNA was subsequently sequenced using the Illumina MiSeq platform (Frasergen Co., Ltd.).

\section{Bioinformatics analysis}

The quality control processing, such as double-end deduplication, splicing and chimera removal, was performed on the raw data after the disembarkation, to obtain highquality valid tags. Then, the sequences obtained above were merged and clustered into operational taxonomic units (OTUs) with $97 \%$ sequence similarity. The sequence with the highest abundance in each classification unit was selected as the representative sequence of the unit (Edgar 
2010). Finally, the sample OTUs were analyzed for abundance, $\alpha$-diversity, $\beta$-diversity, and the bacterial community structure at each classification level.

Table 1 Statistical table for the sequencing volume

Fig. 1 Obeserved species sparse graph. The abscissa represents the sequences randomly selected per sample, and the ordinate represents the number of OTU found at corresponding depth. As the sequencing quantity increased, the number of species found in each sample tended to be flat. Hypophthalmichthys molitrix group (LC1LC3), Aristichthys nobilis group (YC1-YC3), Ctenopharyngodon idellus group (CC1-CC2), and Carassius auratus group (JC1-JC3), Water group (SS, $\mathrm{SZ}, \mathrm{SX})$

\section{Results}

\section{High-throughput data statistics}

For the present study, the Illumina MiSeq platform was used for the paired-end sequencing of DNA fragments. After the initial screening of the original sequencing data, the chimera and question sequences were removed, and the sequence quantity for the subsequent analysis of each sample was obtained. As shown in Table 1, the valid tags of each sample were greater than 40,000. Based on the dilution curve obtained by randomly selecting a certain number of sequencing sequences and the corresponding number of species (Fig. 1), it can be observed from Table 1 and Fig. 1 that as the sequencing quantity increased, the number of species found tended to be flat, indicating that the sequencing depth is enough to reflect the diversity contained in the present samples.

\section{Shared OTU analysis}

The obtained sequences were merged, and the OTUs were divided according to $97 \%$ sequence similarity using the UCLUST sequence comparison tool of the QIIME software (Edgar 2010). A total of 4839 OTUs were obtained. The number of OTUs could represent the richness of the species. Among these, the OTUs detected in the JC, CC, YC, LC and S groups were 2416, 2575, 1915, 1548 and 1015 , respectively.

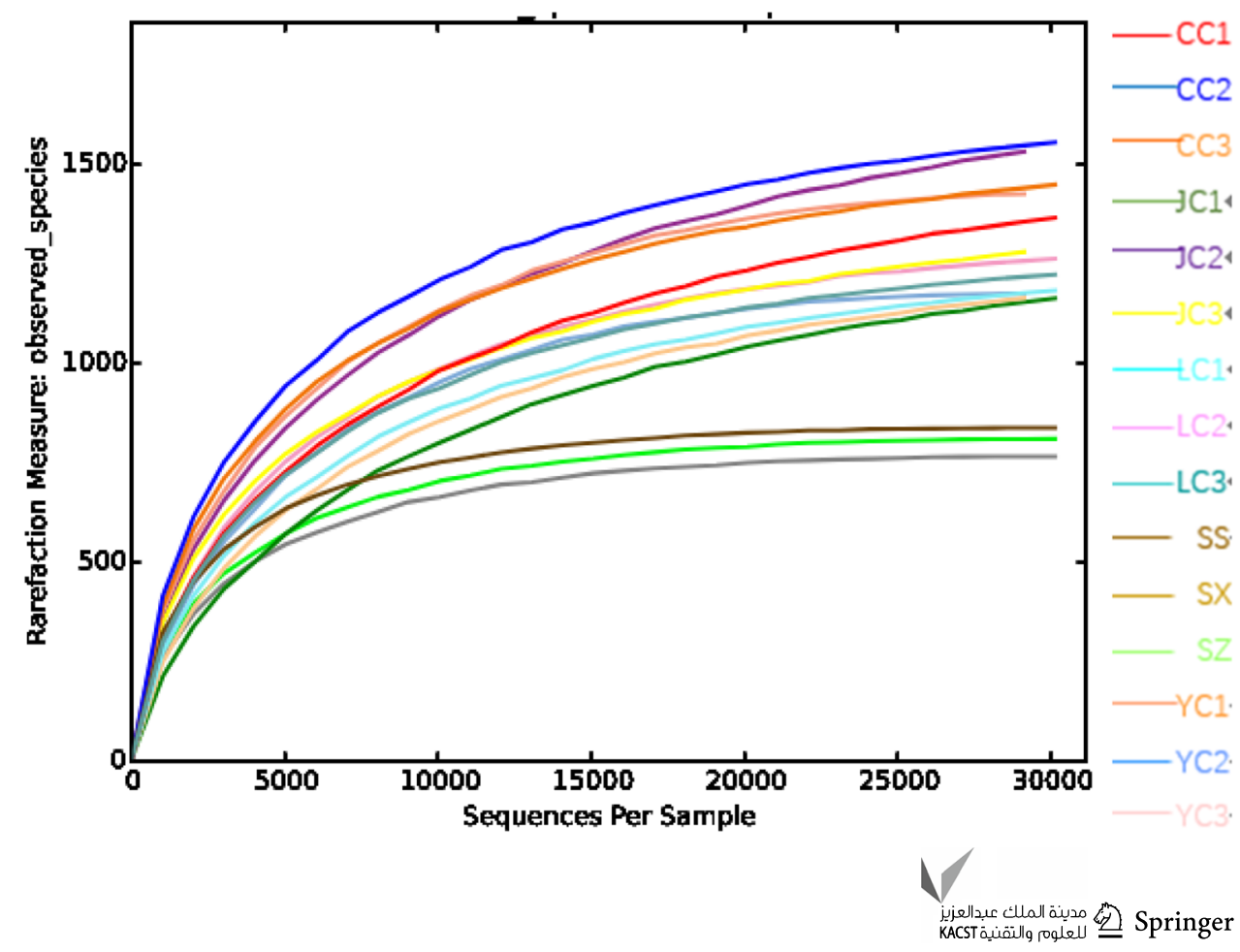


The R software was used to calculate the number of shared OTUs in each group based on the obtained OTU abundance matrix, and the proportion of shared and unique OTUs in each group was visually presented by a Venn diagram. Figure 2 shows that the number of shared OTUs for the five groups was merely 147, and the percentage of unique OTUs in the $\mathrm{CC}, \mathrm{JC}, \mathrm{LC}, \mathrm{YC}$ and $\mathrm{S}$ groups were $29.36,9.56,37.92 \%, 4.54$ and $51.63 \%$, respectively, indicating that half of the OTUs in the water sample can be detected from the intestines of inhabited fishes. At the same time, there were some differences in the unique OTU numbers of these fish groups, and the LC group had the highest unique OTU ratio. From the perspective of the shared OTU in all fish groups, there were relatively more shared OTUs among the CC, JC and YC groups, and there were relatively few shared OTUs between these three groups and the LC group. Furthermore, the unique OTU in the LC group was relatively the highest in the fish intestinal group, indicating that there is a relatively significant difference between the bacteria species in the intestinal contents in the LC group and those in other fish intestinal contents.

\section{The a-diversity analysis}

The Chao1 estimator estimates the number of species that actually exist in the community by counting the number of OTUs detected once and twice in the community (i.e., "Singleton" and "Doubleton"). Unlike Chao1, the Shannon-Wiener index takes into account the richness and evenness of colonies. The higher the Shannon index value, the higher the diversity of the community. It can be observed from the microbiota richness of each group that there was a significant difference (Fig. 3). Group S (water sample group) had the lowest microbiota richness, when compared to the fish group $(P<0.01)$. YC and LC had similar richness $(P>0.05)$, and were lower than JC and CC $(P<0.01)$. The richness in the $\mathrm{CC}$ group was higher than that in the JC group $(P<0.05)$. Therefore, $\mathrm{CC}>\mathrm{JC}>\mathrm{YC}$ and $\mathrm{LC}>\mathrm{S}$ can be considered in terms of microbiota richness. According to the Shannon index in Fig. 4, the diversity of the CC group was significantly higher than that of the other groups $(P<0.05)$, while the diversity of intestinal content samples in the JC, YC and LC groups and environmental samples was not significantly different $(P>0.05)$.
Fig. 2 Venn diagram. The number of each block indicates the number of shared or unique OTUs for the group contained in the block. The shared OTU for the CC and JC groups was 1601 , the shared OTU for the $\mathrm{YC}$ and LC groups was 811, the shared OTU for the CC and YC groups was 1047, the shared OTU for the CC and LC groups was 494, the shared OTU for the $\mathrm{CC}$ and $\mathrm{S}$ groups was 362 , the shared OTU for the $\mathrm{S}$ and JC groups was 226, the shared OTU for the $\mathrm{S}$ and $\mathrm{YC}$ groups was 362, the shared OTU for the YC and JC groups was 1455, and the shared OTU for the LC and JC groups was 528. $L C$ Hypophthalmichthys molitrix group, CC Ctenopharyngodon idellus group, YC Aristichthys nobilis group, JC Carassius auratus group, $S$ water group

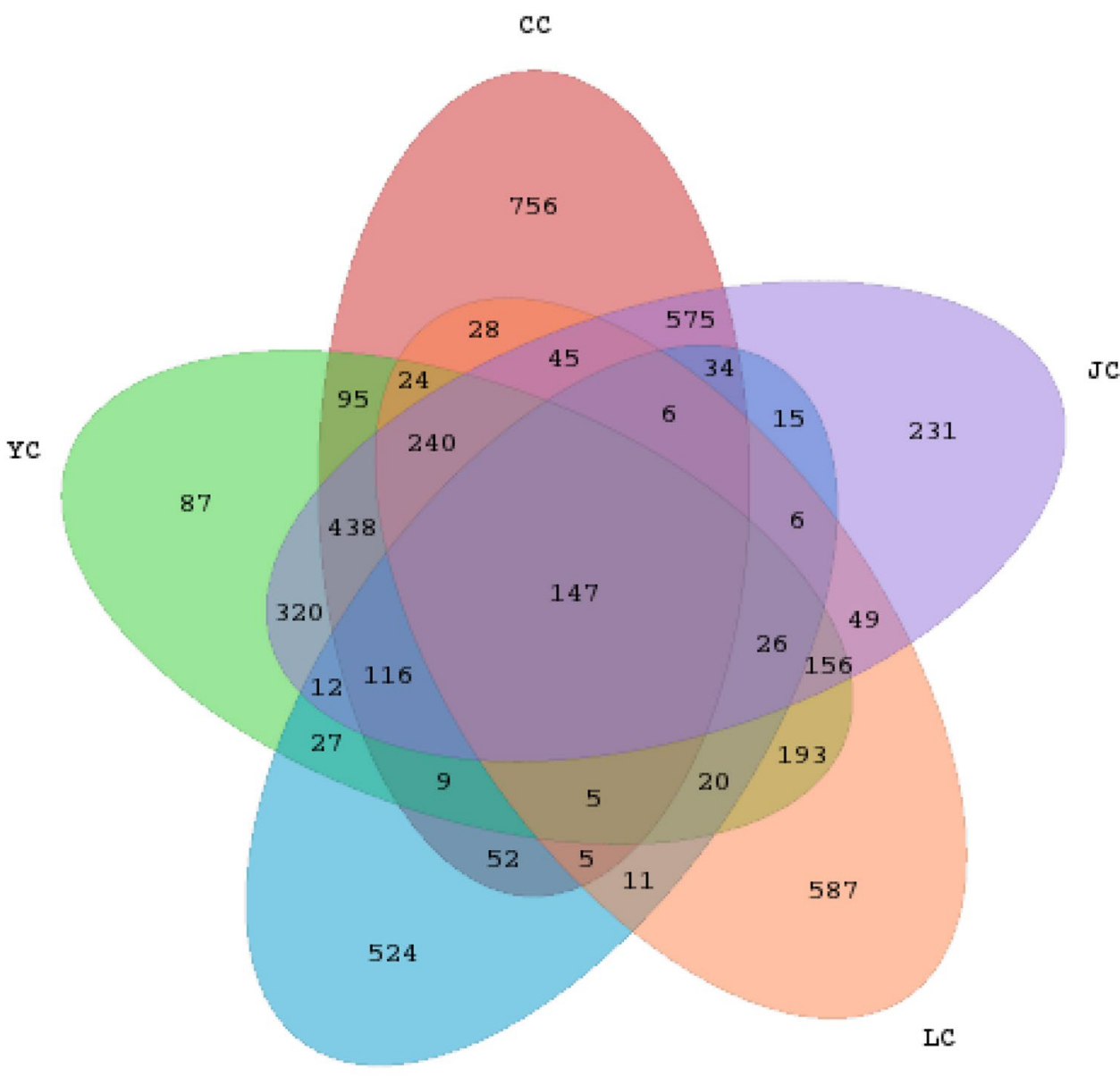


Fig. 3 Chao1 index. The Chao 1 estimator estimates the number of species that actually exist in the community by counting the number of OTUs detected once and twice in the community. The different lowercase letters indicate the significant difference $(P<0.05)$. LC Hypophthalmichthys molitrix group, CC Ctenopharyngodon idellus group, YC Aristichthys nobilis group, JC Carassius auratus group, $S$ water group

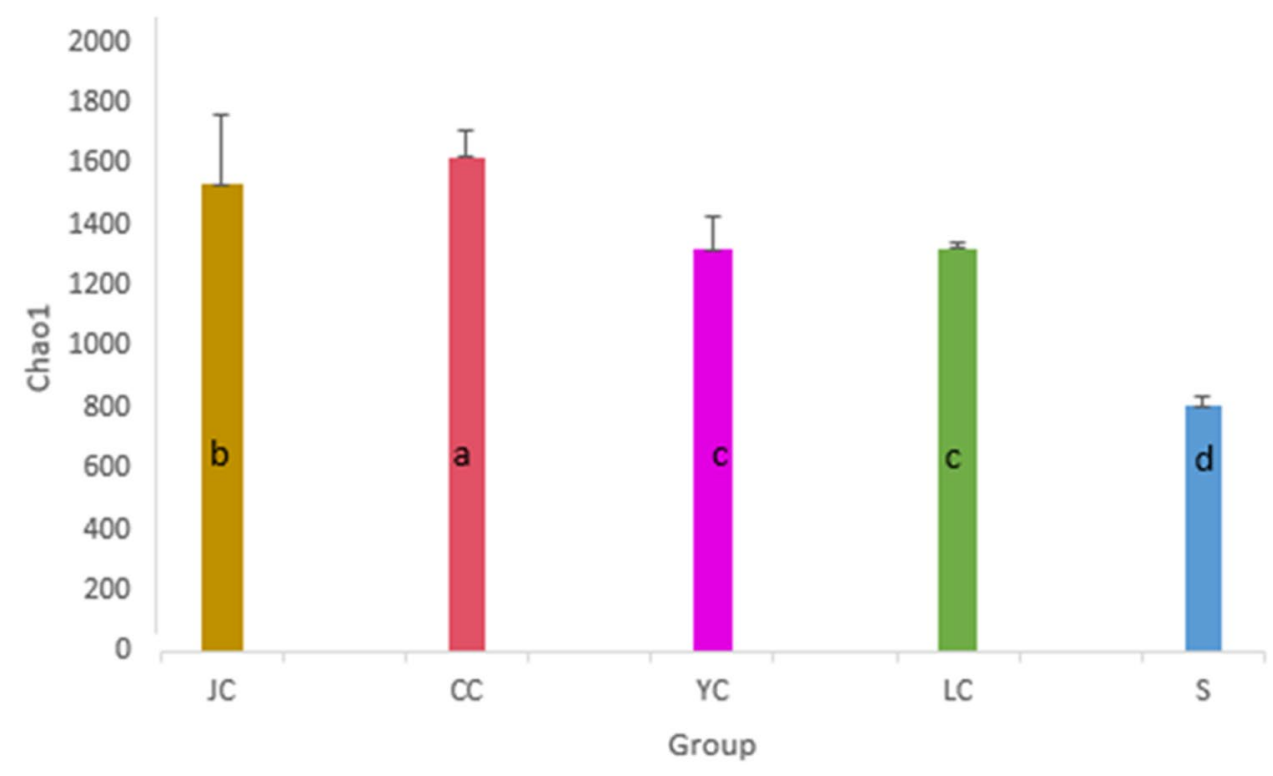

Fig. 4 Shannon diversity index. The higher the Shannon index value, the higher the diversity of the community. The different lowercase letters indicate the significant difference $(P<0.05)$. LC Hypophthalmichthys molitrix group, CC Ctenopharyngodon idellus group, YC Aristichthys nobilis group, JC Carassius auratus group, $S$ water group

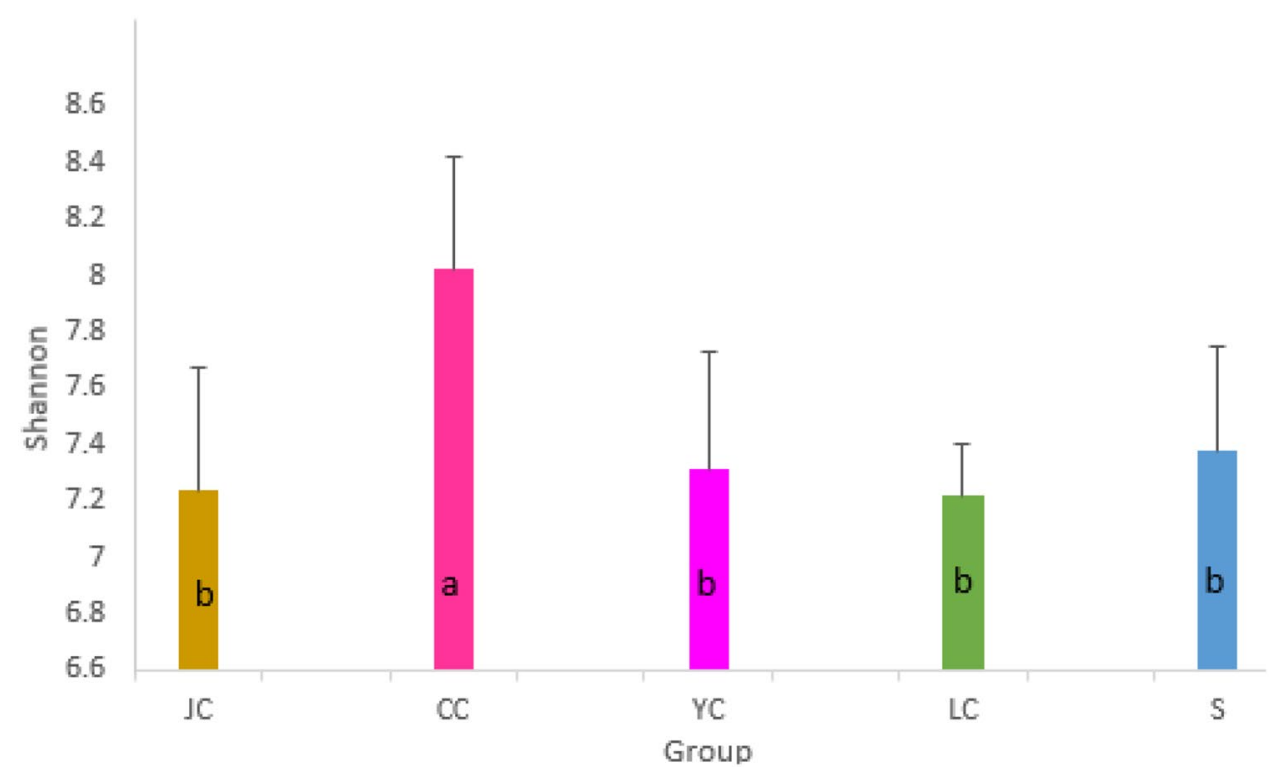

\section{$\beta$-diversity analysis}

The main purpose of the $\beta$-diversity analysis is to determine the similarity of the community structure among different samples (Magali et al. 2013). According to the analysis performed by Unweighted Unifrac NMDS (Fig. 5), the differences within each sample group were small, and these could all gather into a cluster, which can be well-grouped into a ranking chart. The distribution distance between the fish intestinal contents group and water sample group was relatively far, indicating that there are certain differences between the water environment and intestinal content in connection to the bacterial community structure. For the fish intestinal contents group, the $\mathrm{YC}$, JC and $\mathrm{CC}$ groups were closer, and these are basically above the horizontal axis, while the three groups were different from the LC group below the horizontal axis.

\section{Taxonomic composition analysis}

The QIIME software was used to obtain the taxonomic composition and abundance distribution table of each sample at each level. Figure 6 presents the histogram distribution of the microbiota, ranking the top 20 in relative abundance at the phylum level. Figure 6 shows that the fish intestines and water samples mainly included Proteobacteria, Cyanobacteria, Firmicutes, Actinobacteria, Chloroflexi, Verrucomicrobia and Bacteroidetes. Proteobacteria were the highest in abundance in each group, accounting for more than $30 \%$. In comparing the top two relative

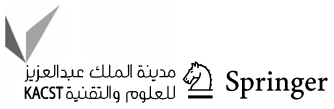


Fig. 5 Unweighted UniFrac NMDS analysis. Each point represents a sample, and points of different colors belong to different samples. The closer the distance between the two points, the higher the similarity of the microbial community structure between the two samples, and the smaller the difference. Hypophthalmichthys molitrix group (LC1-LC3), Aristichthys nobilis group (YC1-YC3), Ctenopharyngodon idellus group (CC1-CC2), and Carassius auratus group (JC1-JC3), Water group (SS, SZ, SX)
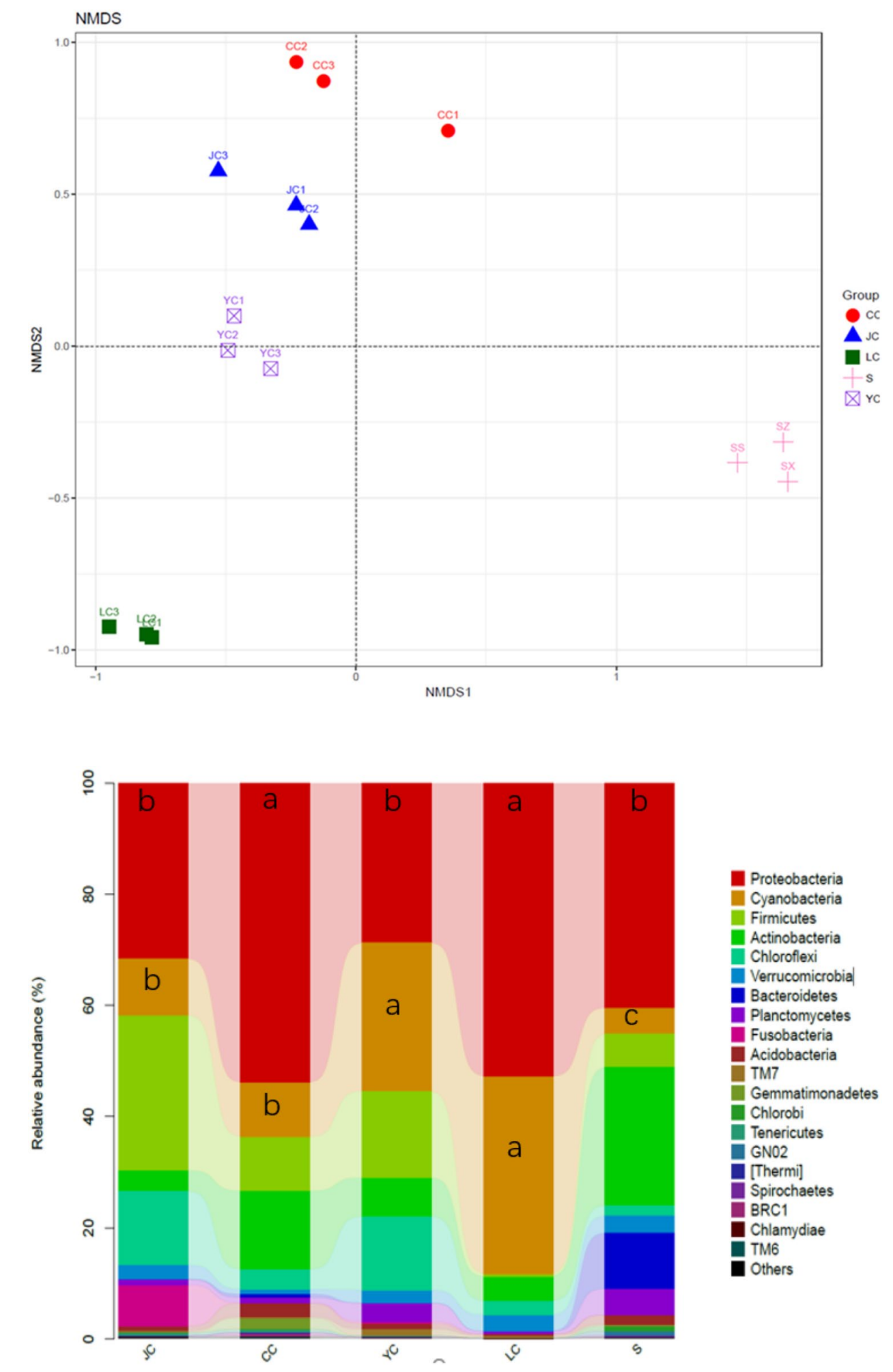

Figure 7 presents the histogram distribution of microbiota, ranking the top 20 in relative abundance at the genus level. The fish intestines and water samples mainly included Synechococcus, Anaerospora, Bacillus, Rhodobacter and Vogesella. In comparing the top two relative abundance genera among
Fig. 6 Bacterial distribution based on the phylum taxonomical level. The different lowercase letters indicate the significant difference $(P<0.05)$. LC Hypophthalmichthys molitrix group, CC Ctenopharyngodon idellus group, YC Aristichthys nobilis group, JC Carassius auratus group, $S$ water group abundance phylum among groups, the relative abundance ratio of Proteobacteria in the $\mathrm{LC}$ and $\mathrm{CC}$ groups was significantly higher, when compared to the other groups $(P<0.05)$, and the LC and YC groups had a higher relative abundance ratio of Cyanobacteria $(P<0.05)$. 
Fig. 7 Bacterial distribution based on the genus taxonomical level. The different lowercase letters indicate the significant difference $(P<0.05)$. $C C$ Ctenopharyngodon idellus group, YC Aristichthys nobilis group, JC Carassius auratus group, $S$ water group

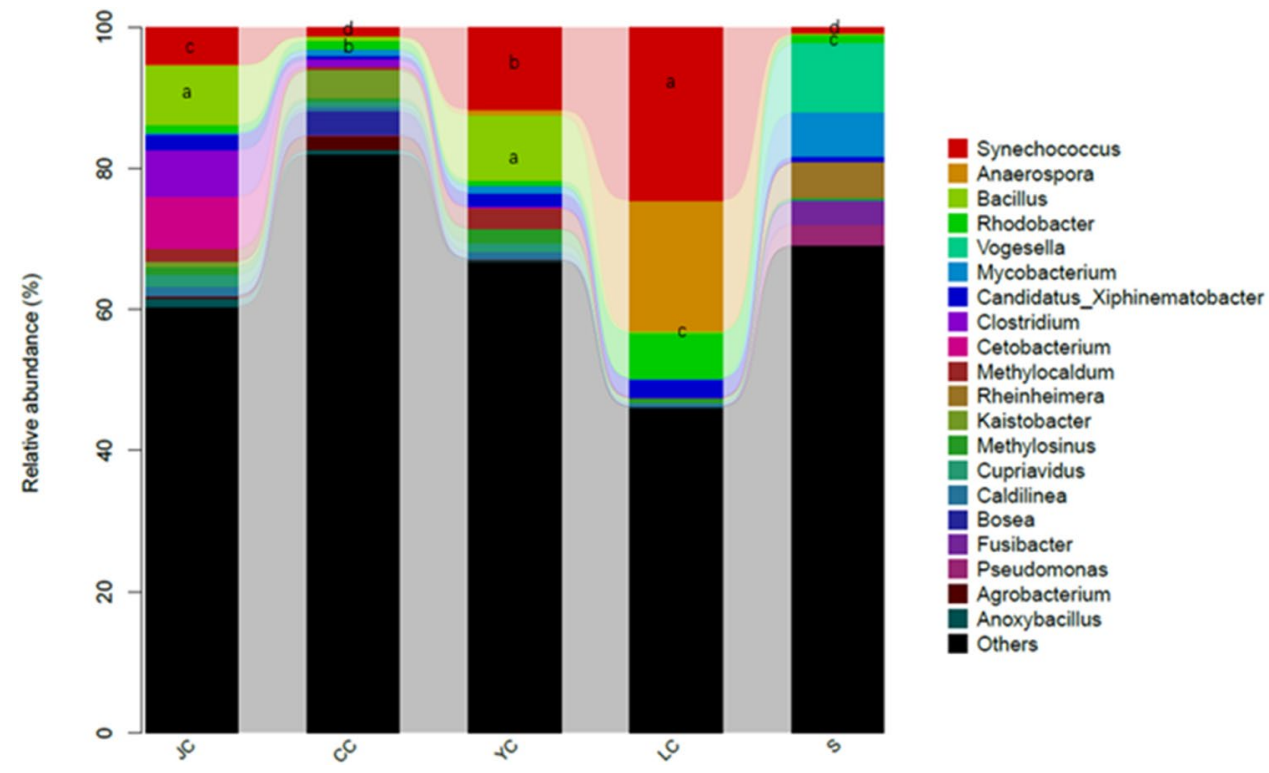

groups, Synechococcus in the LC group had the highest relative abundance $(P<0.05)$, while Bacillus in the YC and JC groups had a relatively high abundance $(P<0.05)$. In the water sample group, the relative abundance of the two genera was the lowest $(P<0.05)$, indicating that the two genera were more suitable for growth in fish intestines.

\section{Taxonomic composition difference analysis between groups}

According to the composition and sequence distribution of each sample at each taxonomic level, the statistical algorithm of Metastats (https://metastats.cbcb.umd.edu/) from the Mothur software was used to determine the absolute abundance difference of the top 10 tax in the genus and phylum level among groups (White et al. 2009). The abundance distribution of the most significant differences among groups is presented in the figure below.

According to Fig. 8, in the fish group, Firmicutes and Methylocaldum were the prevalent taxa in the JC and YC groups $(P<0.05)$. Anaerospora was the prevalent bacterium in the LC group $(P<0.05)$. The prevalent taxa in the water sample group $(P<0.05)$ were Phenylobacterium, Bacteroidetes and Actinobacteria. In conclusion, fish species have different prevalent taxa due to different environments and feeding habits.

\section{Discussion}

Hypophthalmichthys molitrix, Aristichthys nobilis, Ctenopharyngodon idellus and Carassius auratus are the most common fish species under the mixed culturing mode in the Loudi area. Among these, Ctenopharyngodon idellus is an herbivorous fish, Carassius auratus is an omnivorous fish, and Hypophthalmichthys molitrix and Aristichthys nobilis are filter-feeding fish. As comprehensively investigated in the present study, it can be observed that half of the OTUs in the water sample can be detected in fish intestines. The ratio of the number of OTUs shared by Aristichthys nobilis, Ctenopharyngodon idellus and Carassius auratus with the water samples accounted for more than $30 \%$ of the total OTU samples in water samples, while the ratio of the shared OTU of the Hypophthalmichthys molitrix and water samples was only $22 \%$, and the unique OTU in the LC group was relatively the highest in the fish intestinal group. Futher more, it can be seen from the analysis in NMDS analysis, the distance between Hypophthalmichthys molitrix group and water group is relatively farthest. Compared to Aristichthys nobilis, Ctenopharyngodon idellus and Carassius auratus, Hypophthalmichthys molitrix exhibited some differences in microbiota structure and bacterial species. At the same time, the top 10 genera of abundance in fish intestines could also be detected in the water samples, indicating that there is a strong correlation between fish intestinal microbiota and the water environment. Among these, the fish with a weak correlation was Hypophthalmichthys molitrix. From the OTU numbers of each group and the Chaol index and Shannon index, it could be observed that Ctenopharyngodon idellus had the highest microbiota richness and diversity, while the water sample had the lowest richness, indicating that the intestinal microbiota diversity of herbivorous fish is significantly higher than that of omnivorous and filter-feed Fish. These are consistent with the findings reported by some scholars (Larsen et al. 2014; Li et al. 2015; Miyake et al. 2015). From the analysis of the taxonomic composition of

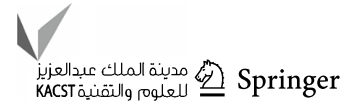


Fig. 8 Abundance distribution of taxa with significant differences among groups. The different lowercase letters indicate the significant difference $(P<0.05)$. The abscissa is the taxon with the most significant difference, and the ordinate is the sequence quantity of each taxon in each group. CC Ctenopharyngodon idellus group, YC Aristichthys nobilis group, JC Carassius auratus group, $S$ water group
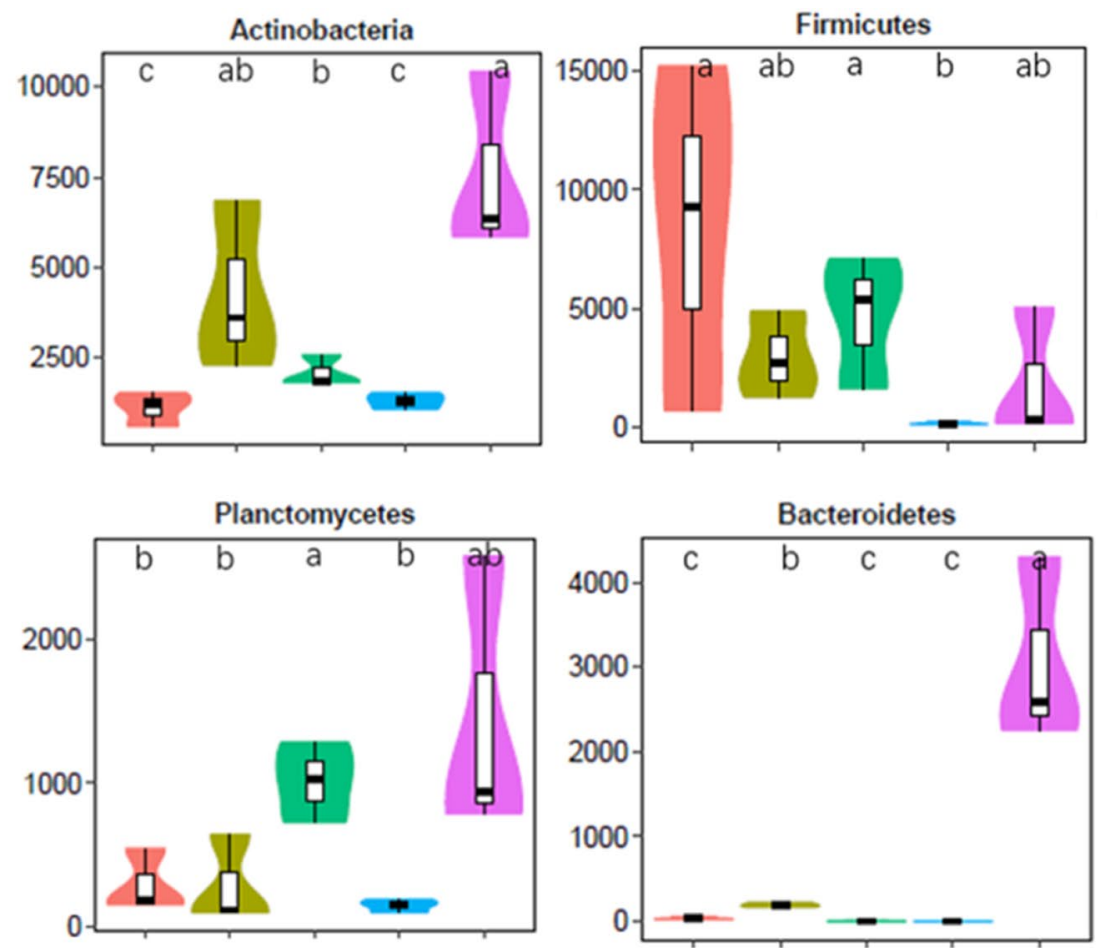

$\mathrm{JC}$
$\mathrm{CC}$
$\mathrm{YC}$
$\mathrm{LC}$
$\mathrm{S}$
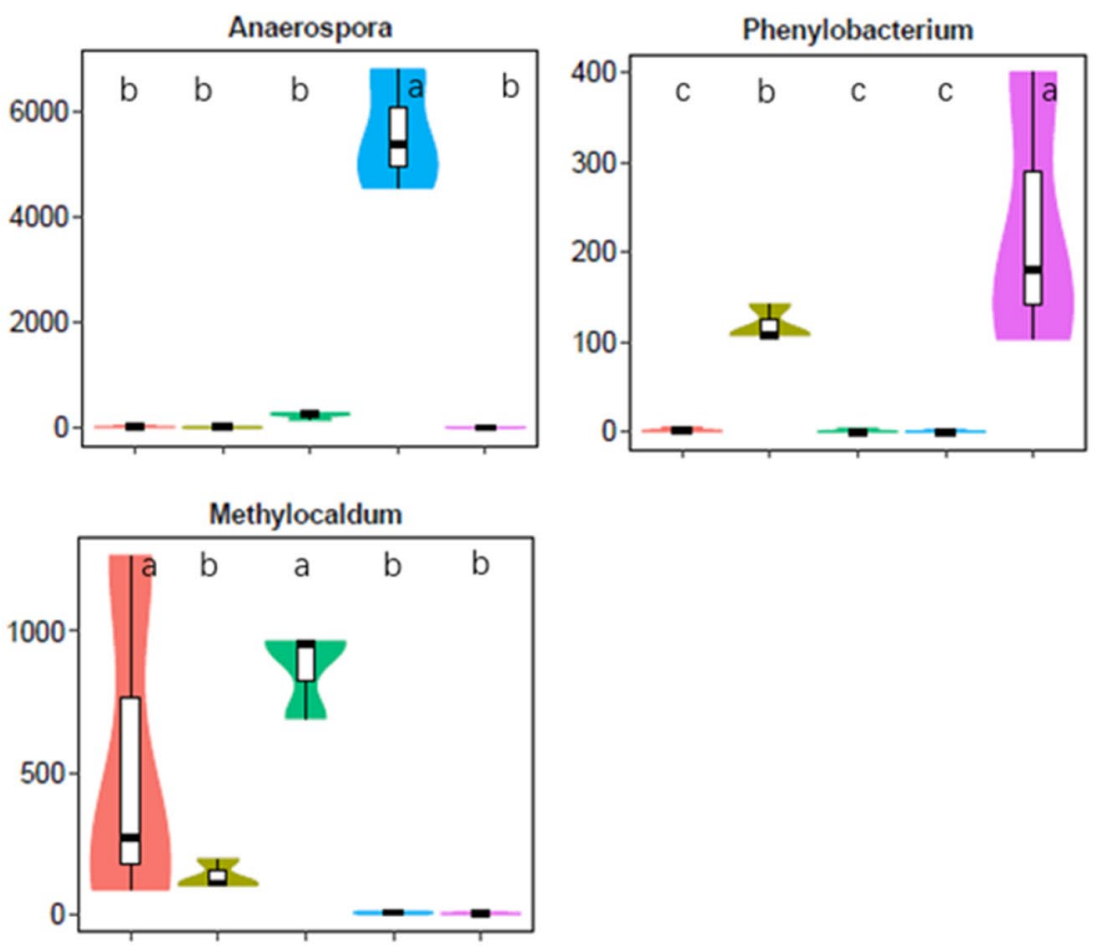

JC CC YC LC $\mathrm{s}$ the phylum, genus and taxonomic composition differences among groups, it can be observed that fish species have different prevalent microbiota. Firmicutes, Methylocaldum and Bacillus are the prevalent taxonomic units in the YC and JC groups. Furthermore, Anaerospora was the prevalent genus in the LC group, and Proteobacteria and Cyanobacteria had higher relative abundance ratios in the $\mathrm{CC}$ group. Moreover, the prevalent taxonomic unit in the water sample group was Phenylobacterium, Bacteroidetes and Actinobacteria. 
In the classification of vertebrates, fish occupies the most important taxonomic status, and has rich ecological diversity (Parma et al. 2016; Ring et al. 2016). According to the results of the present study, there is a large difference in the structure of the intestinal microbiota among fish species. Compared to mammals, the main microbiota of fish gut are more complicated. The effect of the ambient water environment in direct contact with fish on the intestinal microbiota of fish is significantly greater than that in terrestrial animals and human beings. Baits, drugs and additive are also fed directly into the water to affect the growth of the fish. Some intestinal microorganisms can be used as indicator organisms of the environment. Microorganism indicator species play an important role in detecting changes in the environment of aquaculture water. Most studies have shown that the main microbiota at the phylum level in freshwater fish were Proteobacteria, Firmicutes and Actinobacteria (Wang et al. 2018; Talwar et al. 2018; Nayak 2010). The present study revealed that Proteobacteria, Cyanobacteria and Firmicutes were the main microbiota in the fish intestines and water samples. At the same time, most reports have indicated that the main microbiota at the genus level in freshwater fish were Aeromonas, Pseudomonas and Bacillus (Wu et al. 2012; Meng et al. 2018; Li et al. 2013; Liu et al. 2014). However, the present study revealed that Synechococcus, Anaerospora and Bacillus were the main microbiota in freshwater fish and the water samples. The possible reason is that different water environments can cause differences in fish intestinal microbiota. At the same time, it was speculated that Cyanobacteria, Synechococcus and Anaerospora are the indicator microbiota of water samples. It is noteworthy that among the top 20 genera with relative abundance, Cetobacterium was the unique genus in fish intestines, and Vogesella was the unique genus in the water sample. This phenomenon indicates that Cetobacterium is likely to be in fish intestines, and that Vogesella is not suitable for survival in fish intestines.

\section{Accession number}

\section{SRP269121.}

Acknowledgements This work was supported by Grants from the Natural science foundation of Hunan province (no: 2020JJ5322)

\section{Compliance with ethical standards}

Conflict of interest The authors declare that they have no conflict of interests in the publication.

Open Access This article is licensed under a Creative Commons Attribution 4.0 International License, which permits use, sharing, adaptation, distribution and reproduction in any medium or format, as long as you give appropriate credit to the original author(s) and the source, provide a link to the Creative Commons licence, and indicate if changes were made. The images or other third party material in this article are included in the article's Creative Commons licence, unless indicated otherwise in a credit line to the material. If material is not included in the article's Creative Commons licence and your intended use is not permitted by statutory regulation or exceeds the permitted use, you will need to obtain permission directly from the copyright holder. To view a copy of this licence, visit http://creativecommons.org/licenses/by/4.0/.

\section{References}

Chen L, Feng L, Jiang WD et al (2015) Dietary riboflavin deficiency decreases immunity and antioxidant capacity, and changes tight junction proteins and related signaling molecules mRNA expression in the gills of young grass carp (Ctenopharyngodon idella). Fish Shellfish Immunol 45(2):307-320

Edgar RC (2010) Search and clustering orders of magnitude faster than BLAST. Bioinformatics 26(19):2460-2461

Jiang Y, Tang L, Zhang F et al (2016) Identification and characterization of immune-related micro RNAs in blunt snout bream, Megalobrama amblycephala. Fish Shellfish Immun 49:470-492

Larsen AM, Mohammed HH, Arisa CR (2014) Characterization of the gut microbiota of three commercially valuable warmwater fish species. J Appl Microbiol 116(6):1396-1404

Li W, Zhou XQ, Feng L et al (2010) Effect of dietary riboflavin on growth, feed utilization, body composition and intestinal enzyme activities of juvenile Jian carp (Cyprinus carpio var. Jian). Aquac Nutr 16(2):137-143

Li XM, Yan QY, Xie SQ et al (2013) Gut microbiota contributes to the growth of fast-growing transgenic common carp (Cyprinus carpio L.). PLoS ONE 8(5):e64577

Li J, Ni J, Li J et al (2015) Comparative study on gastrointestinal microbiota of eight fish species with different feeding habits. J Appl Microbiol 117(6):1750-1760

Liu WS, Yang YO, Zhang JL et al (2014) Effects of dietary microencapsulated sodium butyrate on growth, intestinal mucosal morphology, immune response and adhesive bacteria in juvenile common carp (Cyprinus carpio) prefed with or without oxidised oil. Br J Nutr 112(1):15-29

Magali NR, Oliver T, Burton et al (2013) A microbiota signature associated with experimental food allergy promotes allergic sensitization and anaphylaxis. J Allergy Clin Immunol 131(1):201-212

Meng XL, Li S, Qin CB et al (2018) Intestinal microbiota and lipid metabolism responses in the common carp (Cyprinus carpio L.) following copper exposure. Ecotox Environ Safe 160:257-264

Mitra A, Mukhopadhyay PK, Homechaudhuris S (2014) Understanding probiotic potentials of Bacills bacterial population isolate from Chiala Chiala (osteoglossiformes; notoperidae) by comparing the enzyme activity in vitro. Int J Pure Appl Zool 2(2):120-127

Miyake S, Ngugi DK, Sting IU (2015) Diet strongly influences the gut microbiota of surgeonfishes. Mol Ecol 24(3):656-672

Nayak SK (2010) Role of gastrointestinal microbiota in fish. Aquac Res 41(11):1553-1573

Nilsson J, Backstrom T, Stien LH et al (2016) Effect of age and rearing environment on genetic parameters of growth and body weight and heritability of skin pigmentation in Arctic Charr (Salvelinus alpinus L.). Aquaculture 453:67-72

Okadinya MO, Ugoh SC, Solomon JR (2013) Studies on the microbial flora found in the intestine of Heteroclarias specie. Academia Arena $5(6): 41-51$

Parma L, Candela M, Soverini M et al (2016) Next-generation sequencing characterization of the gut bacterial community of gilthead sea bream (Sparus aurata L.) fed low fishmeal based diets with increasing soybean meal levels. Anim Feed Sci Technol 222:204-216

Ring E, Zhou Z, Vecino JL et al (2016) Effect of dietary components on the gut microbiota of aquatic animals. A never-ending? Aquac Nutr 22(2):219-282 
Shao H, Zhang C, Wang C et al (2020) Intestinal mucosal bacterial diversity of antibiotic-associated diarrhea (AAD) mice treated with Debaryomyces hansenii and Qiweibaizhu powder. 3 Biotech 10:392. https://doi.org/10.1007/s13205-020-02383-2

Talwar C, Nagar S, Lal R et al (2018) Fish gut microbiome: current approaches and future perspectives. Indian J Microbiol 58(4):397-414

Wang AR, Ran C, Ring $\varnothing$ E et al (2018) Progress in fish gastrointestinal microbiota research. Rev Aquac 10(3):626-640
White JR, Nagarajan N, Pop M (2009) Statistical methods for detecting differentially abundant features in clinical metagenomic samples. PLoS Comput Biol 5:e1000352

Wu SG, Wang GT, Angert ER et al (2012) Composition, diversity, and origin of the bacterial community in grasscarp intestine. PLoS ONE 7(2):e30440 